\title{
Novel Rapid Nondestructive Technique for Locating Tiny Voids in Metallization Line
}

Zheng Hao Gan, and Cher Ming Tan

Division of Microelectronics, School of Electrical \& Electronic Engineering, Nanyang

Technological University, Singapore 639798

A novel nondestructive failure analysis technique to locate tiny voids in narrow copper metallization line rapidly is developed. The metal line can be imbedded in a passivation layer. The principle of the technique is based on the principles of resistance contrast image (RCI) [1] and optical beam induced resistance change (OBIRCH) [2], and electron beam heating is applied. Finite element analysis software, ANSYS, is used to simulate the temperature distribution and current flow in the metal line considering temperature dependent material properties.

FIG. 1 shows the structure for finite element analysis, and FIG. 2 shows the computation results, which will be explained in this work. The sensitivity of the technique in term of void size, void shape, and inter-void distance resolution will be presented as shown in FIG.3. The effect of metal line length is also considered. One can see that the minimum void size that is detectable can be as low as $50 \mathrm{~nm}$, and the shape detectable can be triangular, slit or square. The inter-void distance resolution is as good as $50 \mathrm{~nm}$. However, the sensitivity decreases as the metal length increase.

In conclusion, preliminary simulation demonstrates that the proposed method could be the promising technique to locate small voids rapidly for deep sub-micron metal lines. Experimental validation of the present simulation and further apparatus design will be undertaken in future.

\section{References}

[1] C.A. Smith et al., IEEE Trans. on Electron Devices, ED-33(2) (1986) 282

[2] K. Nikawa et al., Jpn. J. Appl. Phys. 34 (1995) 2260 


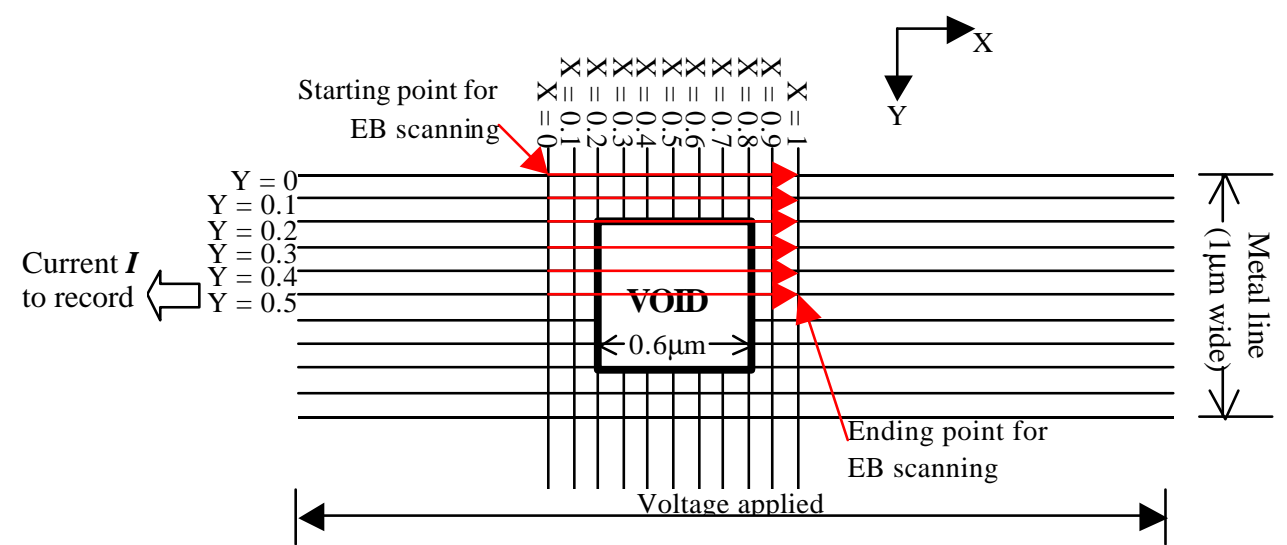

FIG. 1. Structure for FE analysis. The EB is scanned perpendicular to X-Y plane.

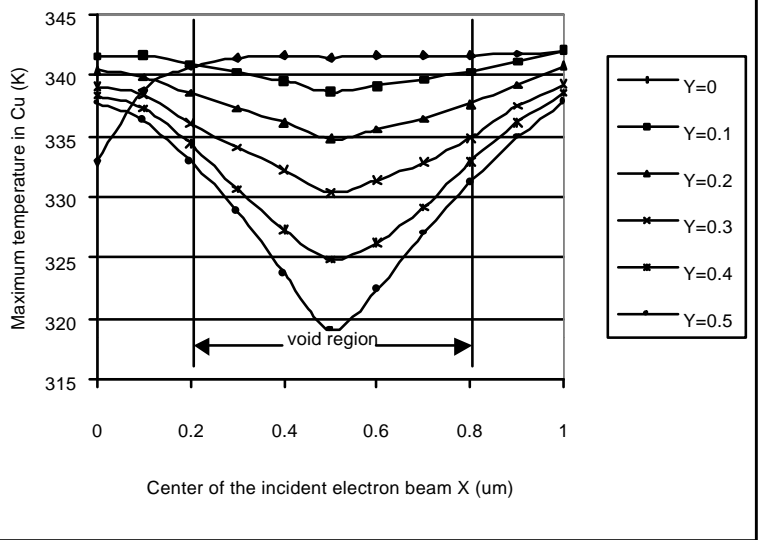

(a)

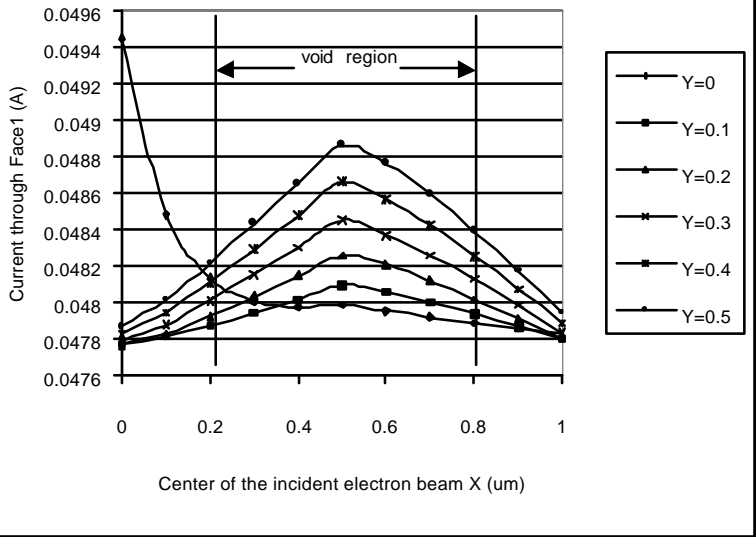

(b)

FIG. 2. (a) Maximum temperature; (b) Current I in the void-line with respect to the EB center

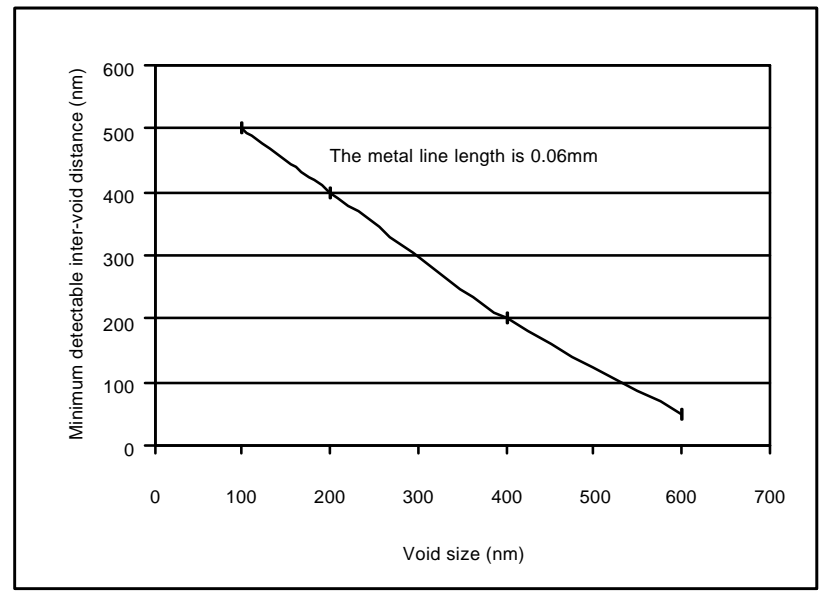

FIG. 3. Minimum detectable inter-void distance vs. void size

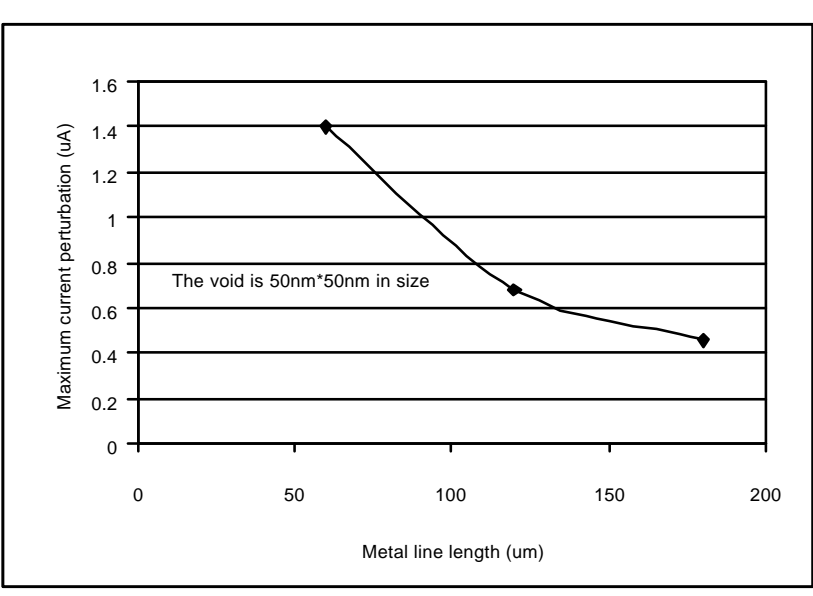

FIG. 4. Maximum current perturbation vs. metal line length 